\title{
EVIDÊnCIAS Sobre AplicaÇão Das Bandas NeURomusCulares Na Reabilitação Do Pé Nos Doentes Pós Acidente Vascular Cerebral
}

\author{
Evidence On The Application Of Neuromuscular Bands In The Rehabilitation \\ Of The FoOt In POST-STROKe PATIENTS \\ EVIDENCIAS SOBRe APLICACIÓn De BANDAS NeUROMUSCULARES EN LA REHABILITACIÓN \\ Del Pie En Pacientes Después De Un Accidente Vascular Cerebral
}

\author{
Raquel Silva ${ }^{1}$; Ana Campos ${ }^{1}$; Eliseu Almeida²; Helena Santos ${ }^{3}$; Sandra Fernandes ${ }^{4}$ \\ 1 - Centro Hospitalar São João; 2 - ARS Norte; 3 - Centro Hospitalar Vila Nova de Gaia/ Espinho; 4 - Centro Hospitalar do Porto
}

\section{RESUMO}

Objetivo: Determinar os benefícios da aplicação das bandas neuromusculares na reabilitação do pé nos doentes pós acidente vascular cerebral.

Metodologia: Estudo de revisão sistemática da literatura segundo o modelo de Joanna Briggs Institute. Incluíram-se estudos em adultos pós acidente vascular cerebral aos quais foram aplicadas bandas neuromusculares. Cinco revisores independentes realizaram a avaliação critica, extração e síntese dos dados.

Apresentação e interpretação dos resultados: Os 4 estudos incluídos reportaram melhorias na postura corporal, deambulação e perceção sensorial.

Conclusão: Os estudos analisados sugerem que as bandas neuromusculares podem ser um método promissor a ser utilizado como método complementar no tratamento do pé do doente pós acidente vascular cerebral, sendo que são necessários mais estudos neste âmbito.

Palavras-chave: acidente vascular cerebral, bandas neuromusculares, Kinesio Taping, pé, reabilitação

\section{RESUMEN}

Objetivo: Determinar los beneficios de la aplicación de bandas neuromusculares en la rehabilitación del pie en pacientes después de un accidente vascular cerebral.

Metodología: Estudio de revisión sistemática de la literatura según el modelo de Joanna Briggs Institute. Se incluyeron estudios en adultos post accidente cerebrovascular a los que se aplicaron bandas neuromusculares. Cinco revisores independientes realizaron la evaluación crítica, extracción y síntesis de los datos.

Presentación e interpretación de los resultados: Los 4 estudios incluidos reportaron mejoras en la postura corporal, deambulación y percepción sensorial.

Conclusión: Los estudios analizados sugieren que las bandas neuromusculares pueden ser un método prometedor que se utilizará como método complementario en el tratamiento del pie del paciente post accidente cerebrovascular, y se necesitan más estudios en este ámbito.

Palabras clave: accidente cerebrovascular, bandas neuromusculares, Kinesio Taping, pie, rehabilitación

\section{ABSTRACT}

Objective: To determine the benefits of the application of neuromuscular bands in the rehabilitation of the foot in post-stroke patients.

Methodology: A systematic review of the literature according to the model of Joanna Briggs Institute. Studies have been included in adults after stroke to which neuromuscular bands have been applied. Five independent reviewers performed the critical evaluation, extraction and synthesis of the data.

Presentation and interpretation of results: The 4 included studies reported improvements in body posture, ambulation and sensory perception.

Conclusion: The studies analyzed suggest that neuromuscular bands may be a promising method to be used as a complementary method in the treatment of the patient's foot post stroke, and further studies are needed in this area.

Key words: stroke, athlétic tape, Kinesio Taping, foot, rehabilitation 


\section{INTRODUÇÃO}

0 acidente vascular cerebral (AVC) é definido como sendo uma interrupção do fornecimento de sangue ao cérebro, geralmente por rutura de um vaso sanguíneo ou obstrução do mesmo devido à presença de um coágulo (1). Como consequência ocorrem danos a nível neurológico, tais como, défices a nível das funções motoras, sensoriais, comportamentais e percetivas.

Para a revisão sistemática da literatura (RSL) que levamos a cabo interessa refletir especificamente sobre as alterações da função motora e sensorial após AVC. Assim, a alteração do tónus, nomeadamente a espasticidade (postura anormal e movimento estereotipado de um membro), requer a nossa atenção pelo risco de desenvolvimento de pé equino, o qual tem impacto sobre a mobilidade (marcha) e sobre o equilíbrio da pessoa. De igual modo, o mecanismo de controlo postural pode ficar afetado no indivíduo que teve um AVC. O seu dia-a-dia é alterado visto que a alteração do controlo postural afeta tarefas básicas como o rolar, sentar, permanecer em pé e andar. Por sua vez, a diminuição da sensibilidade proprioceptiva (postural e vibratória) contribui para a perda da capacidade de executar movimentos eficientes e controlados, para a diminuição da sensação e noção de posição e de movimento, impedindo e diminuindo novas aprendizagens motoras no hemicorpo afetado (2). Dadas as complicações motoras a que o indivíduo está sujeito pós AVC, é deveras importante uma atuação no sentido de evitar a perda de capacidade funcional associada aos défices neurológicos relacionados com a função motora, sensorial e postural.

Perante o exposto, o Enfermeiro Especialista em Enfermagem de Reabilitação (EEER), sustenta a sua tomada de decisão de forma documentada, tendo como foco a qualidade $\mathrm{e}$ os resultados dos cuidados especializados em enfermagem de reabilitação. De acordo com o Regulamento das Competências Específicas do Enfermeiro Especialista em Enfermagem de Reabilitação a área de intervenção é direcionada para a manutenção e promoção do bem-estar e da qualidade de vida, a recuperação de funcionalidade, maximização das capacidades e prevenção de complicações, dirigida à pessoa ao longo do seu ciclo vital (3).

No processo de reabilitação do pé no doente pós AVC, existem várias técnicas de reabilitação disponíveis, sendo que nos últimos anos a aplicabilidade das bandas neuromusculares (BN) ou Kinesio taping (KT) tem ganho mais adeptos e popularidade, tendo vindo a ser desenvolvidos cada vez mais estudos acerca da sua utilidade neste processo.

A Kinesio Taping é uma técnica que envolve a aplicação de uma banda elástica terapêutica sobre a pele, e foi desenvolvida na década de 1970 pelo quiropata japonês Kenzo Kase, com o objetivo de proporcionar ao doente um recurso terapêutico que auxiliasse 0 músculo e outros tecidos a atingirem a sua homeostasia no intervalo entre as sessões de quiropraxia. Alguns autores referem que a técnica baseia-se na indução de estímulos sensoriais adequados sobre a pele, através da aplicação de uma banda elástica, que pode ser utilizado para potencializar e facilitar a homeostasia tecidular e corporal em qualquer condição humana ${ }^{(4)}$. Tendo sido desenvolvida para facilitar o processo de cura natural do corpo, e ao mesmo tempo fornecer suporte e estabilidade aos músculos e articulações sem restringir a amplitude de movimento do corpo, tem sido usada para tratar uma variedade de condições ortopédicas, neuromusculares, neurológicas e médicas.

As bandas neuromusculares podem atuar no músculo, nas articulações, na circulação linfática, nas fáscias, na derme, em tendões e ligamentos. Assim, os benefícios advogados pelas bandas neuromusculares podem ser subdivididos em quatro grandes funções: função dérmica, função muscular, função articular e função linfática. Contudo, pode-se, de acordo com o manual da técnica, estimular ou inibir determinado músculo, utilizando-se as bandas neuromusculares. A aplicação da banda neuromuscular feita no sentido da inserção para a origem do músculo (de distal para proximal) teria efeito inibitório e, quando o sentido de aplicação da banda neuromuscular é da origem para a inserção do músculo (de proximal para distal), teria efeito de facilitação ou estimulação muscular (4).

Segundo Kenzo Kase (2003) esta terapêutica tem como objetivos: aliviar a dor e as sensações anormais da pele e músculos; proporcionar maior equilíbrio e suporte aos músculos durante os movimentos; diminuir edemas linfáticos e sanguíneos; corrigir os desalinhamentos articulares e biomecânicos; criar mais espaço nos níveis epidérmicos, dérmicos e hipodérmico; promover estímulos e melhorar a propriocepção ${ }^{(5)}$.

Apesar da aplicação das bandas neuromusculares na área da neurologia, ser cada vez mais uma realidade, tal como já referido anteriormente, a verdade é que até à data poucos estudos foram realizados para validar o uso das mesmas, nesta população. Os efeitos terapêuticos advogados pela aplicação das bandas neuromusculares continuam controversos e não existe evidência científica que os sustente. Assim, tendo vindo, nos últimos anos, a ganhar relevo a sua aplicação na área da neurologia, interessa clarificar e validar esta técnica no processo de reabilitação do doente pós AVC ao nível do pé. Isto é, averiguar quais as evidências sobre aplicação das bandas neuromusculares na reabilitação do pé no doente pós AVC.

\section{MÉTODO}

A Revisão Sistemática seguiu as orientações do Joanna Briggs Institute $(\mathrm{JBI})$. Foram definidos e aplicados critérios de seleção segundo a metodologia PEO que define, segundo Mendes et al (2008) a População, a Exposição e os resultados, do inglês Outcome (14). Participantes: todos os adultos, com diagnóstico de AVC; Exposição: aplicação das bandas neuromusculares no pé; Outcomes: reabilitação. Apenas foram selecionados ensaios clínicos randomizados.

Durante o mês de julho de 2018 efetuou-se a pesquisa nas subsequentes bases de dados científicas eletrónicas: Medline ${ }^{\circledR}$ e CINAHL $₫$, definindo-se como critérios de inclusão os estudos publicados em português, inglês e espanhol, com texto de acesso livre 
e data de publicação de 2012 a 2018 e com idade igual ou superior a 19 anos (all adults).

\section{Estratégia de pesquisa e identificação dos estudos}

Inicialmente, foi realizada uma pesquisa limitada à base de dados Medline ${ }^{\circledR}$ e CINAHL $®$ utilizando as frases booleanas, ((MH "Stroke+") AND ((MM "Athlétic tape") OR ("kinesio tap")) AND (MM "Foot") AND (MH "Rehabilitation+")) e ((MH "Stroke+") AND ((MH "Tapes+") OR (MM "Athlétic Tape") OR ("kinesio tap*")) AND (MH "Foot+") AND (MM "Rehabilitation")), respetivamente. Devido à existência de diferenças nos processos de indexação nas bases de dados bibliográficas, optou-se para além do uso de vocabulário controlado (descritores), pela utilização do termo livre "Kinesio Taping" na pesquisa. Com essa estratégia, houve uma recuperação de um número maior de referências, garantindo a identificação da maioria dos trabalhos publicados dentro dos critérios pré-estabelecidos.

Seguiu-se uma seleção através da leitura dos títulos e resumos. A seleção de artigos foi realizada de forma individual por 3 revisores (AC, EA e RS) com consenso de mais 2 revisores (HS e SF).

\section{Avaliação da qualidade metodológica dos estudos}

A qualidade metodológica foi avaliada por 3 revisores (AC, EA e RS) independentes com consenso de outros 2 revisores (HS e SF) usando o instrumento de avaliação Critical Appraisal Checklist for Randomised Controlled Trials, para ensaios clínicos randomizados da JBI ${ }^{(6)}$, em que foram considerados estudos de qualidade os que obtivessem até, no máximo, 3 respostas negativas (definido, em consenso prévio à análise dos investigadores, de modo a preservar um score igual ou superior a $75 \%$ de respostas positivas). Para classificação do nível de evidência dos estudos recorreu-se aos níveis de evidência de Hockenberry, tendo os estudos sido classificados com nível de evidência lb (que corresponde a evidência obtida de pelo menos um ensaio clínico) ${ }^{(7)}$.

\section{Extração de dados}

Os dados foram extraídos por 3 revisores (AC, EA, RS) e com consenso de outros 2 revisores (HS e SF), de forma independente, entre julho e agosto de 2018, usando o instrumento de Joanna Briggs Institute data extraction form for systematic review of experimental/observational studies e incluiu as características dos participantes, as características da intervenção, os métodos de estudo e os resultados pertinentes dos outcomes avaliados.

\section{Síntese dos dados}

A síntese dos dados foi realizada nos meses de julho e agosto de 2018, com o objetivo de sumariar a narrativa dos dados. Nela participaram 3 revisores (AC, EA, RS), com o consenso de outros 2 revisores (HS e SF). Para tal elaborou-se uma tabela para cada um dos estudos incluídos na revisão sistemática, e nas quais constaram: título, autor, país, ano, tipo de estudo, população, método, objetivos, resultados e conclusões.

\section{Apresentação dos resultados}

Foram identificados 1219 artigos: 911 da CINAHL $®$ Plus with Full Text (ESBCO ${ }^{8}$ - host via ESSSM) e 308 da MEDLINE $®$ with Full Text $(\mathrm{ESBCO} \otimes$ - host via ESSSM). Destes, 850 foram excluídos pelos limitadores de pesquisa, ficando 235 artigos da CINHAL $®$ e 134 da MEDLINE $®$. Com apoio do Endnote ${ }^{\circledR}$, e após compilação dos artigos mencionados anteriormente, verificaram-se 33 artigos duplicados. Desses, 330 foram excluídos pelo título, visto focarem locais anatómicos diferentes do delineado para esta revisão; e um foi excluído após leitura do resumo. Os 5 estudos selecionados foram avaliados metodologicamente, após aplicação da Checklist for Randomized Controlled Trials da JBI, sendo que 4 estudos foram considerados válidos uma vez que a percentagem de respostas verdadeiras foi superior a 75\% (tal como preconizado anteriormente) e 1 excluído por não conferir a validade metodológica estabelecida pelos investigadores. Após esta seleção individual e de forma a aumentar a confiabilidade e transparência do processo de seleção, reuniram-se todos os investigadores, tendo-se constatado unanimidade da seleção dos estudos.

Dos 4 artigos incluídos, 3 encontram-se escritos em língua inglesa e 1 em língua portuguesa, datados de 2015 e 2017. Um estudo foi publicado na Turquia, um no Brasil, um na República da Coreia e um no Irão. Todos os estudos respeitam os critérios éticos referentes ao processo de investigação realizado. Relativamente aos tipos de estudos, os 4 são quantitativos.

$\mathrm{Na}$ tabela 1, são apresentados os estudos que compuseram esta Revisão Sistemática, e dela consta: os autores dos estudos, o ano de publicação, o país, as características dos participantes, as intervenções e os resultados dos estudos incluídos, bem como, o nível de evidência (N.E) de cada estudo e avaliação metodológica (A.M).

\section{RESULTADOS E DISCUSSÃO}

$\mathrm{Na}$ análise particular dos estudos selecionados verificou-se uma grande heterogeneidade dos mesmos, relativamente aos objetivos, ao método, ao tipo de amostra e à forma como estudam a ação das bandas neuromusculares quando aplicadas ao pé nos doentes pós AVC.

Da análise dos resultados dos estudos incluídos nesta RSL, podemos inferir que a aplicação das bandas neuromusculares teve efeitos positivos ao nível do equilíbrio, do controlo motor do pé, da perceção, da amplitude do tornozelo e na marcha.

No estudo 1 os autores revelam que após implementação do plano terapêutico que consistiu na aplicação das bandas neuromusculares no pé, há uma melhoria da perceção, com consequências na melhoria do equilíbrio dos indivíduos do grupo ${ }^{(8)}$. 


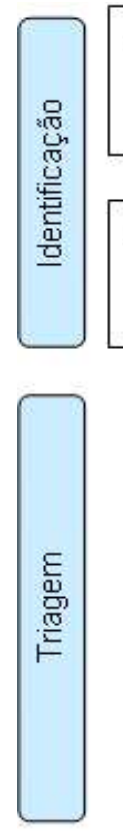

Artigos identificados através da pesquisa na base de dados CINHAL $(\mathrm{R})(\mathrm{n}=911)$

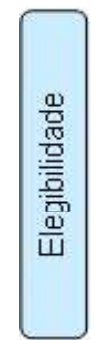

Figura 1 - Diagrama do processo de seleção
Artigos identificados através da pesquisa na base de dados MEDLINE(R) $(n=308)$

Artigos adicionaisidentificados por outras fontes $(\mathrm{r}=0)$.

\section{Filtros:}

- Data de publicaçã̃o: 2012-2018

- Língua: inglês, espanhol, português - Idade: all orilts
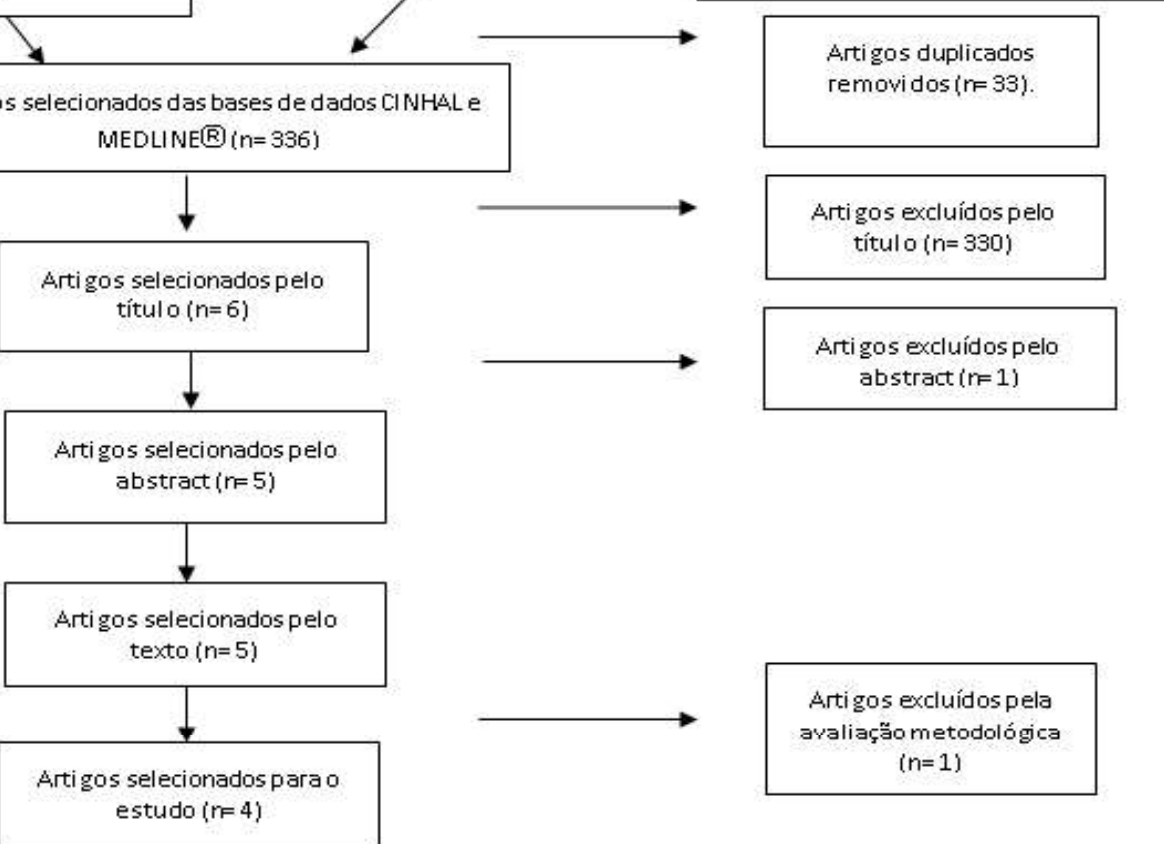

Artigos excluídos pelo abstract ( $r=1)$

Artigos excluídos pela avaliação metodológica ( $n=1)$

\begin{tabular}{|c|c|c|c|c|c|}
\hline $\begin{array}{c}\text { Artigo }\left(\mathrm{n} .^{\circ}\right), \text { Autor, } \\
\text { Ano (País) }\end{array}$ & $\begin{array}{l}\text { Tipo de Estu- } \\
\text { do/População }\end{array}$ & Intervenções & $\begin{array}{l}\text { Resultados/ } \\
\text { Outcomes }\end{array}$ & N.E. & A.M. \\
\hline $\begin{array}{l}\text { 1, Yazici, Guclu- } \\
\text { Gunduz, Bayraktar, } \\
\text { Aksoy, Nazliel, } \\
\text { Kilinc, Yildirim, } \\
\text { Irkec, } 2015 \\
\text { (Turquia) }^{(8)}\end{array}$ & $\begin{array}{l}\text { Ensaio clínico } \\
\text { randomizado }\end{array}$ & $\begin{array}{l}\text { Experimental: } 19 \text { pessoas com AVC } \\
\text { Controlo: } 16 \text { pessoas saudáveis sem } \\
\text { alterações neurológicas }\end{array}$ & $\begin{array}{l}\text { Melhorias no equilíbrio dos indivíduos do } \\
\text { grupo experimental que se associa ao } \\
\text { aumento da perceção somatosensorial } \\
\text { devido à presença de bandas } \\
\text { neuromusculares no pé. }\end{array}$ & lb & $10 / 13$ \\
\hline $\begin{array}{l}\text { 2, Lima, Wanderley, } \\
\text { Henriques, Filho, } \\
\text { Oliveira, } 2017 \\
\text { (Brasil) }^{(9)}\end{array}$ & $\begin{array}{l}\text { Ensaio clínico } \\
\text { randomizado e } \\
\text { duplo cego }\end{array}$ & $\begin{array}{l}\text { Experimental: } 7 \text { pessoas com AVC aos } \\
\text { quais foram aplicadas bandas } \\
\text { neuromusculares com tensão } \\
\text { Controlo: } 7 \text { pessoas com AVC aos } \\
\text { quais foram aplicadas bandas } \\
\text { neuromusculares sem tensão }\end{array}$ & $\begin{array}{l}\text { Não foi comprovada a hipótese de que a } \\
\text { aplicação de bandas neuromusculares } \\
\text { com tensão era eficaz no ganho de } \\
\text { amplitude articular do tornozelo. No } \\
\text { entanto } 3 \text { pessoas do grupo de controlo } \\
\text { afirmaram sentir diferença positiva } \\
\text { durante utilização das bandas } \\
\text { neuromusculares ao nível da marcha e } 2 \\
\text { pessoas referiram diferença positiva ao } \\
\text { nível da perceção. }\end{array}$ & $\mathrm{lb}$ & $12 / 13$ \\
\hline $\begin{array}{l}\text { 3, Yang, Heo, Lee, } \\
2015 \text { (República da }^{(10)} \\
\text { Coreia) }\end{array}$ & $\begin{array}{l}\text { Estudo } \\
\text { experimental } \\
\text { primário } \\
\text { randomizado }\end{array}$ & $\begin{array}{l}\text { Experimental: } 19 \text { pessoas com AVC aos } \\
\text { quais, foram aplicadas bandas } \\
\text { neuromusculares e posteriormente } \\
\text { foram avaliados o equilíbrio postural } \\
\text { e a distribuição da pressão no pé } \\
\text { Controlo: } 19 \text { indivíduos com AVC } \\
\text { foram submetidos aos mesmos } \\
\text { procedimentos do grupo } \\
\text { experimental mas em ordem inversa. }\end{array}$ & $\begin{array}{l}\text { Verificou-se alteração do centro de } \\
\text { equilíbrio após aplicação das bandas } \\
\text { neuromusculares. }\end{array}$ & lb & $10 / 13$ \\
\hline $\begin{array}{l}\text { 4, Rojhani-Shirazi, } \\
\text { Amirian, } \\
\text { Meftahi, } \\
2015 \text { / Irão (11) }\end{array}$ & $\begin{array}{l}\text { Ensaio clínico } \\
\text { controlado } \\
\text { randomizado. }\end{array}$ & $\begin{array}{l}\text { Experimental: } 20 \text { pessoas com AVC } \\
\text { aos quais foram aplicadas bandas } \\
\text { neuromusculares } \\
\text { Controlo: } 20 \text { pessoas com AVC aos } \\
\text { quais não foram aplicadas bandas } \\
\text { neuromusculares }\end{array}$ & $\begin{array}{l}\text { Melhoria estatisticamente significativa } \\
\text { no equilíbrio e no apoio do pé no chão } \\
\text { nos indivíduos do grupo experimental em } \\
\text { comparação com o grupo de controlo. }\end{array}$ & $\mathrm{lb}$ & $10 / 13$ \\
\hline
\end{tabular}


No estudo levado a cabo por Lima et al (estudo 2), os achados não foram significativos ao nível do ganho da amplitude do tornozelo. Porém, os autores relatam que os participantes do estudo referiram melhoria ao nível da marcha, bem como da perceção ${ }^{(9)}$.

Relativamente ao estudo 3, após a aplicação das bandas neuromusculares o grupo experimental mostrou alterações do centro do equilíbrio ${ }^{(10)}$.

0 estudo 4 menciona melhoria estatisticamente significativa ao nível do equilíbrio e do controlo motor do pé (11).

Perante os dados acima enumerados é possível verificar que após colocação das bandas neuromusculares são descritos os seguintes ganhos: no equilíbrio (estudos 1 e 4); na alteração no centro de equilíbrio (estudo 3); na perceção (estudos 1 e 2); no controlo do pé (estudo 4) e na marcha (estudo 2). Apesar de haver relação dos ganhos após colocação de bandas neuromusculares nos estudos a sua comparação é questionável devido à discrepância existente no desenho dos estudos, na sua metodologia e por vezes informação oculta, nomeadamente: tipo de AVC e respetiva área de lesão; tempo de recuperação pós AVC, cuidados prévios à aplicação das bandas neuromusculares, método de aplicação das bandas neuromusculares detalhado; tempo de exposição; escalas e método de avaliação de resultados.

Aos estudos selecionados para esta Revisão Sistemática foi atribuído grau $\mathrm{C}$ quanto ao grau de recomendação segundo a qualidade da evidência científica, de acordo com a escala de Jovell e Navarro-Rubio (12). Visto que existe uma insuficiente evidência científica, a decisão de adotar a tecnologia deve basear-se em outros critérios.

Esta Revisão Sistemática da Literatura traz como principal contribuição à prática dos enfermeiros de reabilitação o conhecimento existente na aplicação das bandas neuromusculares no pé do doente pós AVC. Por outro lado, orienta para uma elaboração mais precisa de novos estudos visto mencionar quais as limitações encontradas nos estudos existentes, bem como algumas sugestões.

\section{CONCLUSÕES}

O AVC tem um grande impacto no ciclo vital da pessoa, visto afetar de forma aguda a sua saúde e qualidade de vida. A enfermagem é uma ciência que se relaciona com as experiências humanas de transição, em que a saúde e o bem-estar se destacam como resultados da intervenção; no entender desta teórica o desafio do profissional de enfermagem em reabilitação centra-se no entendimento do processo de transição, desenvolvendo estratégias adaptativas que ajudem a pessoa a recuperar a estabilidade e o bem-estar ${ }^{(13)}$.

Neste sentido, cabe ao enfermeiro especialista em reabilitação implementar programas de reabilitação baseando-se na melhor evidência existente, de forma a obter ganhos que se traduzam na recuperação/minimização dos défices do doente pós AVC. Ao realizarmos a presente RSL verificámos que existem alguns benefícios na aplicação das bandas neuromusculares na reabilitação do pé no doente pós AVC, nomeadamente ao nível da postura corporal, marcha e perceção sensorial. Apesar das limitações encontradas, das quais constam o número reduzido de estudos, o tamanho reduzido da amostra e as diferenças metodológicas que dificultam a comparabilidade dos resultados, não existiu compromisso no atingimento dos objetivos inicialmente traçados.

Portanto, consideramos que seria proveitosa a realização de mais estudos nesta área, tendo em conta um maior número de participantes, um follow-up maior, uma descrição mais detalhada e monitorizada da aplicação das bandas neuromusculares, bem como, a realização de um programa de reabilitação de forma a complementar esta técnica.

\section{REFERÊNCIAS}

1. OMS. Stroke, Cerebrovascular accident [serial on the Internet]. 2018 [cited 2018 jul 10]: Available from: http://www.who.int/topics/cerebrovascular_accident/en/

2. Bobath, Berta. Hemiplegia em Adultos Avaliação e Tratamento. 3. ${ }^{a}$ ed. São Paulo: Editora Manole; 2001

3. OE. Regulamento das Competências Específicas do Enfermeiro Especialista em Enfermagem de Reabilitação. Lisboa. 2010

4. Kase, K., Lemos, T., Dias, E. “Kinesio taping® - Introdução ao método e aplicações musculares". 2. ${ }^{a}$ Ed. São Paulo: Livraria e Editora Andreoli; 2013

5. Kase, K., Wallis J., Kase, T. Clinical Therapeutic Applications of the Kinesio Taping Method. Albuquerque, New México: Kinesio Taping Association. 2003

6. Joanna Briggs Institute. Joanna Briggs Institute reviewers' manual: 2014 edition. Australia: The Joanna Briggs Institute; 2014

7. Hockenberry, M., Wilson, D., \& Barrera, P. Implementing evidencebased nursing pratice in a Pediatric Hospital. Pediatric Nurs. 2006; 324., 371-377.

8. Yazici, G. S., Guclu-Gunduz, A. S., Bayraktar, D. S., Aksoy, S. S., Nazliel, B. S., Kilinc, M. S., Irkec, C. S. Does correcting position and increasing sensorial input of the footand ankle with Kinesio Taping improve balance in stroke patients? NeuroRehabilitation. 2015; 345-353.

9. Pereira Lima, D. L., Wanderley, D. S., de Miranda Henriques, L. F., de Moura Filho, A. G., \& de Oliveira, D. A.. Efeitos do kinesio taping na dinâmica articular durante a marcha de pacientes após acidente vascular encefálico. Fisioter Bras. 2017; 29-37.

10. Sung Rae, Y. S., Seo Yoon, H. E., \& Hee Jae, L. E. 2015 Immediate effects of kinesio taping on fixed postural alignment and foot balance in stroke patients J Phys Ther Sci.2015; 3537 3540.

11. Rojhani-Shirazi, Z., Amirian, S., \&amp; Meftahi, N. Effects of Ankle Kinesio Taping on Postural Control in Stroke Patients. J Stroke Cerebrovasc Dis. 2015; 2565-2571.

12. Jovell AJ, Navarro-Rubio MD. Evaluación de la evidencia científica. Med Clin Barc 1995; 105: 740-743.

13. MCEwen, M., \& Wills, E. M. Bases Teóricas de Enfermagem. Porto Alegre: Artmed. 2016

14. Mendes KDS, Silveira RCDCP, Galvão CM. Revisão integrativa: método de pesquisa para a incorporação de evidências na saúde e na enfermagem. Texto \& Context Enferm. 2008;174.:758-64. 